\section{Extensive Necrotizing \\ Fasciitis after Fat \\ Grafting for Bilateral \\ Breast Augmentation: \\ Recommended Approach and Management}

Chong Han Pek ${ }^{1}$, Jane $\mathrm{Lim}^{1}$, Hui Wen $\mathrm{Ng}^{1}$,

Han Jing Lee ${ }^{1}$, Wei Chen Ong ${ }^{1}$, Anthony Tun Lin Foo ${ }^{2}$, Chwee Ming Lim ${ }^{3}$, Mark Thong ${ }^{3}$,

Sandeep Jacob Sebastin ${ }^{2}$, Thiam Chye Lim

${ }^{1}$ Division of Plastic Reconstructive and Aesthetic Surgery, Department of Surgery, ${ }^{2}$ Department of Hand and Reconstructive Microsurgery, University Orthopaedics, Hand \& Reconstructive Microsurgery Cluster, ${ }^{3}$ Department of Otolaryngology-Head and Neck Surgery, National University Health System, Singapore

Correspondence: Jane Lim

Division of Plastic Reconstructive and Aesthetic Surgery, Department of Surgery, National University Health System, 1E, Kent Ridge Road, Singapore 119228, Singapore

Tel: +65-6779-5555, Fax: +65-6775-0913

E-mail: cfslimj@nus.edu.sg

No potential conflict of interest relevant to this article was reported.

Received: 20 Dec 2014 • Revised: 3 Jan 2015 • Accepted: 19 Jan 2015 pISSN: 2234-6163 • elSSN: 2234-6171

http://dx.doi.org/10.5999/aps.2015.42.3.365 • Arch Plast Surg 2015;42:365-367

Copyright (C) 2015 The Korean Society of Plastic and Reconstructive Surgeons

This is an Open Access article distributed under the terms of the Creative Commons Attribution Non-Commercial License (http://creativecommons.org/licenses/by-nc/3.0/) which permits unrestricted non-commercial use, distribution, and reproduction in any medium, provided the original work is properly cited.

Fat grafting is widely recognised to be an ideal treatment for volume and contour defects. Its appeal lies in the fact that it results in long-lasting enhancement of the breast without the use of alloplastic material. Common complications include

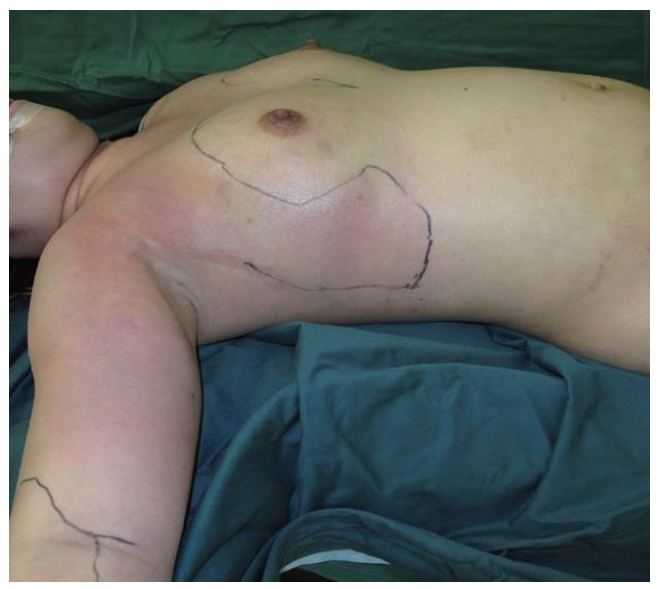

fat resorption, calcifications, nodular formation, infective complications such as abscesses and sepsis, and less than ideal surgical outcomes $[1,2]$. The actual incidence of necrotizing fasciitis (NF) associated with fat grafting is not known [3]. There are few case reports of severe infection and NF after fat grafting, and all required urgent surgical treatment and antibiotic therapy [3-5]. We present a case report of extensive NF after fat grafting for bilateral breast augmentation and discuss the subsequent management of the case.

The patient was a 27-year-old Chinese female who presented with hypotension, fever and pain, and redness and swelling over both breasts. Ten days prior to her presentation, she underwent bilateral liposuction of the thighs, liposuction of the abdomen, and bilateral fat grafting to the breasts. Her procedure was performed in a different country; hence, details about the procedure and operating surgeon were not available. One day after surgery, she developed fever and bilateral breast pain. She was given paracetamol by her operating surgeon and no antibiotic therapy was initiated. Over the next five days, her symptoms progressed to both upper arms and the affected skin became more erythematous, swollen, and painful. On examination, the patient was febrile and in septic shock. Extensive erythema with swelling was present over her chest wall extending into both upper arms with palpable subcutaneous crepitus (Figs. 1, 2).

With the clinical diagnosis of NF, aggressive fluid resuscitation and inotropes were started to address the septic shock. Empirical intravenous penicillin G, clindamycin and ceftazidime were initiated in accordance to our institutional protocol. Urgent computed tomography scans revealed ill-defined

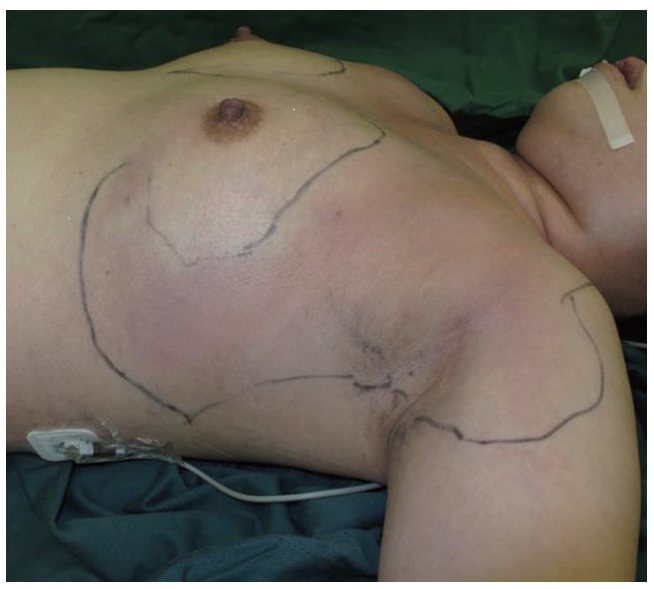

Fig. 2.

Clinical pictures of necrotizing fasciitis in the left breast and upper arm.

Fig. 1.

Clinical pictures of necrotizing fasciitis in the right breast and upper arm. 


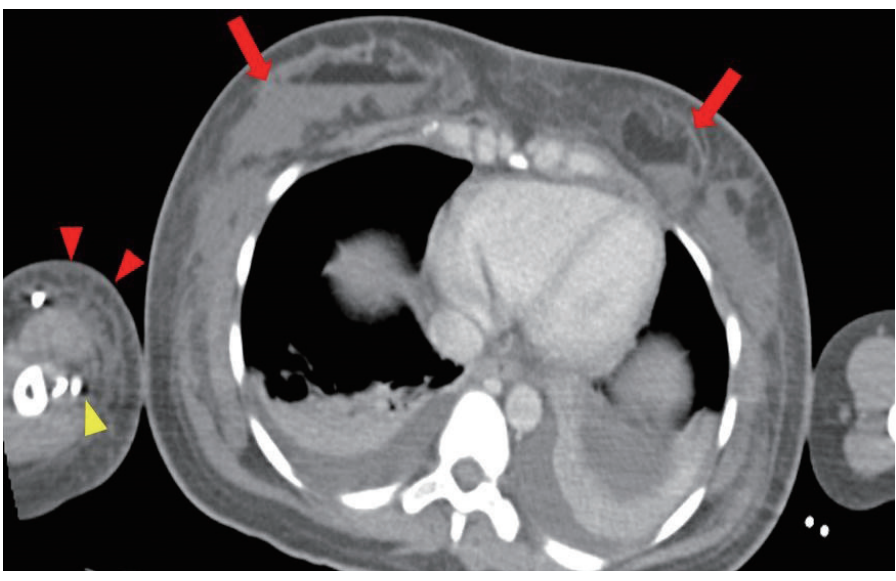

Fig. 3.

Computed tomography scan of the thoracic region. These contrast-enhanced axial images of the thorax show ill-defined masses in the anterior thorax within the breast parenchyma as well as the sub-glandular plane, indicated with red arrows. There were also fat-fluid levels within these masses. These findings were suggestive of fat necrosis with secondary infection as the fat within these masses had likely liquefied; the fluid component in this context was likely to be pus. In addition, there was extensive subcutaneous

fat stranding and oedema over the right upper arm region, shown by the red arrowheads. Hypodensities around the contrast enhanced vessels of the right upper limb, shown with yellow arrowhead, may represent gas formation within tissue planes consistent with necrotizing fasciitis. These findings were corroborated during surgery.

masses within the breast parenchyma and subglandular planes of the chest wall as well as large collections containing an air-fluid level (Fig. 3).

The patient proceeded immediately to surgery and debridement; $620 \mathrm{~mL}$ of foul-smelling "dishwater fluid" admixed with necrotic fat was evacuated from the right sub-glandular plane extending into the right upper arm and $435 \mathrm{~mL}$ of fluid with the same consistency was drained from the left sub-glandular plane extending into the left axilla (Fig. 4). The overlying skin was not necrotic and bilaterally the breast tissue was still viable.

A repeat debridement 24 hours after the initial operation was planned, and further unhealthy tissue was removed. The planned third debridement was extended superiorly into the neck, due to the involvement of the left sternocleidomastoid muscle.

The patient's sepsis gradually improved with adequate debridement and washout, antibiotics, and supportive therapy in the intensive care unit. Bacterial cultures grew Proteus mirabilis, Enterobacter aerogenes, Enterococcus faecalis, and Bacteroides fragilis. Antibiotic therapy was adjusted to piperacillin-tazobactam according to culture sensitivities. The patient required another four debridement operations before all wounds were closed and a skin graft was placed on

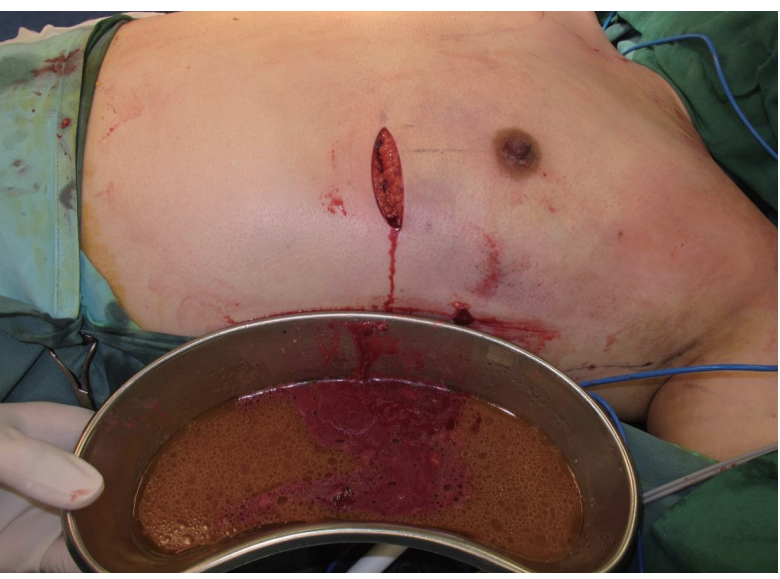

Fig. 4.

Intraoperative photo showing the drainage of pus and necrotic quefied fat from the left breast.

the right arm. The patient continues to receive regular follow-up for the management of her hypertrophic scars (Fig. 5).

A task force reviewing fat grafting found that the overall complication rate of fat grafting was not unduly high and that cases of severe complications and demise were very rare. In addition, the authors acknowledged the rarity of cases of severe infection, sepsis, and septic shock [1]. Another recent systematic review reported that $0.7 \%$ of patients ( 10 of 1,453$)$ experienced minor infections and $2 \%$ of patients ( 35 of 1,453$)$ experienced major infections [2]. The minor infections required outpatient oral antibiotic therapy, whereas the major infections often required surgical drainage and inpatient antibiotic therapy. The major infections were predominantly a result of injecting large boluses of fat $[2,4]$.

Devastating infections, as described in our case, are rare. Our tenets of managing such a case would include: (1) the early diagnosis and identification of the planes involved, (2) aggressive antibiotic therapy, (3) an early multidisciplinary approach and operative debridement, and (4) frequent planned relook debridements.

The suspicion for NF in this patient's scenario would be high due to the recent surgical intervention, in which multiple planes of soft tissue had been breached. The presence of non-vascularised fat grafts within multiple tracks and planes of injection led to a greater spread of the infection. Computed tomography imaging, in this instance, was crucial for establishing the extent of involvement and ensuring that the appropriate multidisciplinary teams were involved early in the patient's care. Preoperative 


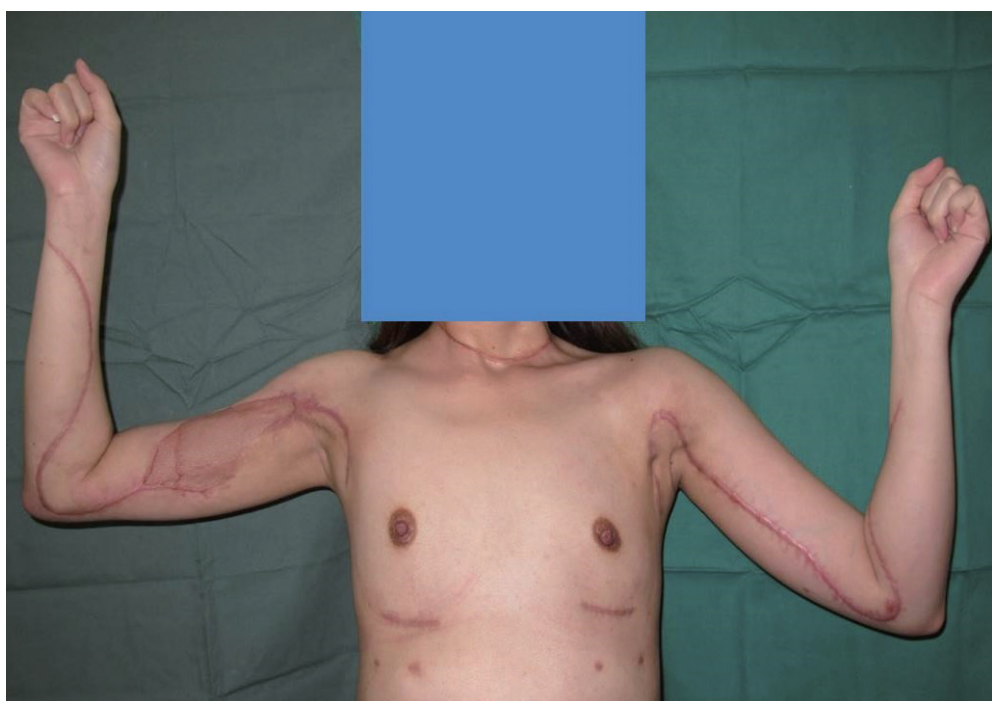

Fig. 5.

Clinical picture of the patient six months after surgery. This is a clinical picture of the patient showing the scars and surgical approach for the debridement of the extensive necrotizing fasciitis in both breasts, arms, and neck. Early diagnosis and treatment allowed us to preserve most of the overlying skin to allow primary closure after the complete debridement of necrotic subcutaneous fascia and tissue; only a small area over the right proximal arm measuring approximately $8 \times 3 \mathrm{~cm}$ required skin grafting for eventual wound closure. imaging also allows for planning the surgical approach and the placement of incisions to maximise surgical exposure and preserve vital tissue while minimising scarring and morbidity.

Antibiotic therapy serves to reduce systemic bacterial load but it does little in the way of source control. Hence, the mainstay of management of NF is expedient and systematic surgical debridement. Reexamination along with repeated wound lavage and debridement every 24 to 48 hours is the treatment of choice until the wound is clean enough for closure [3].

In our patient's case, we postulated that the cause of infection leading to NF was multifactorial. Fat grafting with large boluses, poor surgical dissection technique, haematoma formation, lack of sterility, and lack of postoperative wound care and follow-up may all have contributed to our patient's severe infection. It was difficult to establish a definitive cause for the infection without additional information about the initial surgery. Fortunately, early diagnosis and treatment limited disease progression in our patient, minimising morbidity and mortality.

In conclusion, with the growing popularity of medical tourism, surgical complications of this nature may present challenges to plastic surgeons more frequently. In such cases, the lack of details about the initial procedure presents a peculiar challenge in itself. In the postoperative patient, the likely cause of infection is multifactorial, and a high index of suspicion is required to diagnose NF early. We feel that computed tomography scans are a crucial component of the management of NF in such cases. Early operative debridement, aggressive antibiotic therapy, and frequent planned relook debridements are important in order to improve patient outcomes and minimise morbidity.

\section{References}

1. Gutowski KA; ASPS Fat Graft Task Force. Current applications and safety of autologous fat grafts: a report of the ASPS fat graft task force. Plast Reconstr Surg 2009;124:272-80.

2. Largo RD, Tchang LA, Mele V, et al. Efficacy, safety and complications of autologous fat grafting to healthy breast tissue: a systematic review. J Plast Reconstr Aesthet Surg 2014;67:437-48.

3. Sherman JE, Fanzio PM, White H, et al. Blindness and necrotizing fasciitis after liposuction and fat transfer. Plast Reconstr Surg 2010;126:1358-63.

4. Talbot SG, Parrett BM, Yaremchuk MJ. Sepsis after autologous fat grafting. Plast Reconstr Surg 2010;126:162e-164e.

5. Lee KS, Seo SJ, Park MC, et al. Sepsis with multiple abscesses after massive autologous fat grafting for augmentation mammoplasty: a case report. Aesthetic Plast Surg 2011;35:641-5. 